\title{
Research status and Prospect of integrated energy system collaborative control based on energy Internet
}

\author{
Peng $\mathrm{Ye}^{1, *}$, Keming $\mathrm{Qu}^{1}$, Feng Sun ${ }^{2}$, Mingli Zhang ${ }^{3}$, and Na Zhang ${ }^{3}$ \\ ${ }^{1}$ Shenyang Institute of engineering, Shenyang 110136, China \\ ${ }^{2}$ State Grid Liaoning Electric Power Supply Co.LTD, Electric Power Research Insitute, Shenyang 110006, China \\ ${ }^{3}$ State Grid Liaoning Electric Power Company Limited Economic Research Institute, Shenyang, 110065, China
}

\begin{abstract}
As the only way to build the energy Internet, the key and difficult point of integrated energy system is to plan it reasonably and efficiently. This paper analyzes and summarizes the basic strategy, key technologies, control difficulties and influencing factors of integrated energy system control, summarizes the existing problems at home and abroad, and summarizes the content framework and control model of general integrated energy system control covering the source, network, load and storage links in a comprehensive way based on the latest research progress. According to the latest research progress, the paper analyzes the current control schemes, and concludes the development trend of the current integrated energy system collaborative control, makes a reasonable outlook on the future, and provides a way of thinking for people to study the integrated energy system in the future.
\end{abstract}

\section{Introduction}

Compared with the traditional single energy system, the advantages of integrated energy system (IES) lie in the complementary advantages of system operation and design. Operation: promote the integration of local renewable and sustainable energy, and reduce pollutant emission by improving the reliability and energy efficiency of the whole system. Design: because of the characteristics of the IES module division area, the energy storage and exchange module is very adaptable. To achieve these, it is undoubtedly very important to optimize the IES.

At present, the research on system control and operation optimization has been quite perfect at home and abroad. Literature [1] uses MILP programming model to design a building level comprehensive energy system, and determines the type of main equipment and installed capacity. The paper introduces the system environmental indicators into the control plan, and quantitatively analyzes the influence of system environment on the control cycle life of IES. The paper proposes two-layer framework structure suitable for the optimal design of distributed energy system in China, including the selection of comprehensive energy equipment type and equipment capacity based on load change. This paper systematically analyzes and summarizes the existing literature, and the main work is shown in Figure 1.

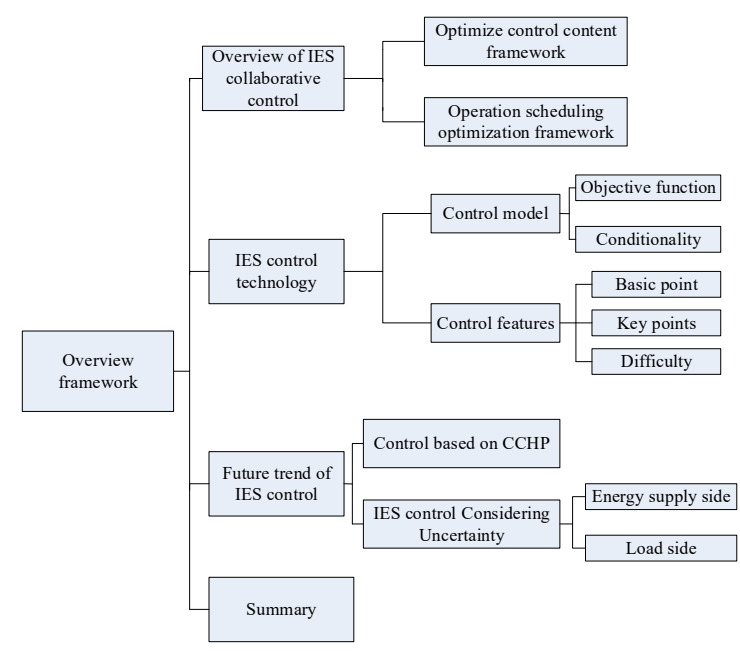

Figure 1. Review of collaborative control

\section{Collaborative control framework}

Specifically, the collaborative control of IES can be regarded as two levels of control problems, the first level is system control, and the second level is operation scheduling control. And the operation scheduling optimization of the second level technically serves the IES system control of the first level, and the optimization results provide sufficient basis for the system control. Therefore, the operation scheduling optimization of IES can be regarded as a sub control process of the system control, as shown in Figure 2. 


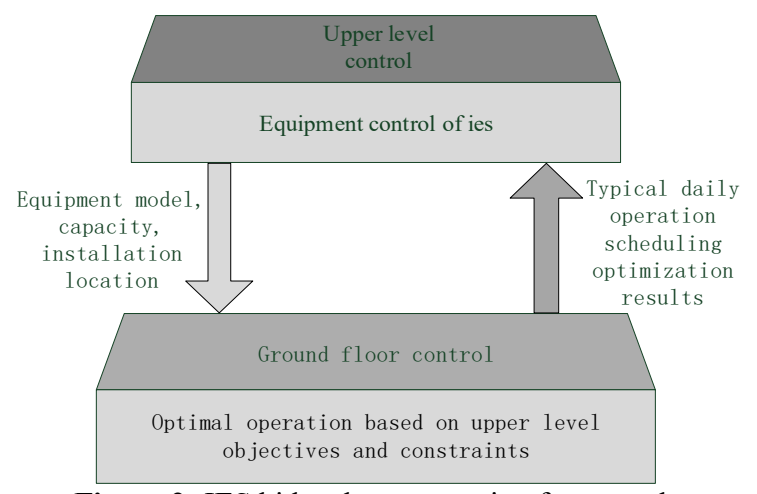

Figure 2. IES bi level programming framework

\subsection{System optimization}

\subsubsection{Operation scheduling optimization}

The research on this level requires that all kinds of scheduling models should be based on the typical daily hourly load demand curve to obtain the typical daily operation scheduling optimization results of each equipment in different energy demand periods, and serve as an important index in IES optimization control.

\subsubsection{System optimization control}

This layer requires different control models based on the typical daily hourly load demand curve in different energy demand periods, and the typical daily optimization results are used as the indicators in IES optimization to provide services for IES optimization. The joint optimization operation in literature [2] is mainly concentrated on the energy supply side, and the combined resource category is relatively single. Literature [3] is further extended to the joint control of electric energy and thermal energy, and the negative impact on the environment is also minimized.

\subsection{Control features of IES}

The following summarizes the previous multi-objective control schemes and their advantages and disadvantages, as well as the schemes now widely used:

(1) Multi objective integer programming. Reference [4] proposed a multi-objective optimization model for large-scale clean power generation control of energy system.

This kind of control scheme adopts the subjective assumption of combining multiple objectives into one measurement, which simplifies the complexity of control, but it will lead to the difficulty of confirming the weighting factors and economic indicators.

(2) Multi objective Fractional Programming

The objective is the ratio of two functions. By comparing the objective of each aspect with its original value, it can provide unbiased measurement of the system and make up for the shortcomings of integer programming. In reference [5], fractional programming is applied to transportation and production management, and good results are achieved. In reference [6], integer variables and uncertain input problems in fractional programming have not been solved perfectly.

(3) Mixed integer fractional programming

At the same time, it has the advantages of integer programming and fractional programming, which can not only solve the ratio optimization problem, but also help to dynamically analyze the capacity expansion plan of power generation facilities, and better reflect the complexity of the energy system. Literature [7] shows that this scheme can help to determine the schemes needed for resource allocation and facility expansion in various complex situations, and then support the longterm control of energy system. This is also a widely used control scheme.

Multi Scenario Control:

The core of multi scenario control is to establish different control scenarios based on natural resource attributes and local load demand, so as to boundary various uncertain factors and effectively reduce the uncertainty and complexity of control problems.

Firstly, the load and output curve analysis based on Control scenario is helpful to improve the prediction accuracy of cold / heat / power demand. Secondly, based on different control scenarios, the off-design characteristics of each equipment in different modes are analyzed, and the constraints of different working scenarios are obtained. Finally, based on the evaluation and verification of the control results of multi scenario control, the rationality and feasibility of the control scheme can be comprehensively analyzed, and effective suggestions are put forward.

Control difficulties:

(1) Coupling of complex energy sources

There are great differences in the physical characteristics of various energy systems. The research on the coupling mode between energy systems and the establishment of relevant mathematical models are the basis and key to realize the complementary operation and control of various energy systems.

(2) Dynamic scalability

The system is constantly developing and changing. It needs to reflect the dynamic index of system capacity, and determine the necessary energy productivity with the corresponding expansion capacity.

\section{IES control model}

The control of IES can be divided into two frameworks, the upper framework of system optimization control and the lower framework of operation scheduling optimization. All kinds of objective functions and constraints are established for these two frameworks.

\subsection{Objective function}

Due to the limitation of optimization algorithm and other objective reasons, the current control scheme usually contains only two or three objective functions, such as economy. Environmental protection, safety and reliability, etc. 
(1) Economy: the control of IES should be based on the whole life cycle of the system.

$$
F_{E}=\sum_{i}\left(B_{i}+Y \cdot F_{i}-R_{i}\right)+Y \sum_{k} D_{k} \cdot O_{k}^{E}
$$

In the above formula, $B_{i}$ is the initial investment cost of the energy storage equipment, $F_{i}$ is the annual operation and maintenance cost of the energy storage equipment, $D_{k}$ is the number of days in the energy demand period in 1 year, $O_{k}^{E}$ is the energy demand standard Typical daily operating cost, $Y$ is the engineering life of the whole system, $R_{i}$ is the Residual value of energy supply and storage equipment.

(2) Environmental protection: usually, the $\mathrm{C}$ emission of the system is used as the measurement index, which is composed of two parts: (1) the emission carried by the input energy itself; (2) the emission generated in the process of energy conversion within the system.

$$
F_{e n v}=Y \sum_{k} D_{k} \cdot O_{k}^{e n v}
$$

In the above formula, $O_{k}^{e n v}$ is the typical daily operating cost under environmental protection criteria.

(3) Reliability: in the basis of economy and environmental protection, the reliability of the system is considered, which is of great significance to the actual control and operation of IES. The energy shortage cost is used to describe the stability of the system.

$$
C_{e n s}=N \sum_{i}^{N} \sum_{t=1}^{24}\left(L_{c u t, i}^{e} \cdot c_{i}^{e}+L_{c u t, t}^{h} \cdot c_{i}^{h}\right)
$$

In the above formula, $N$ is the number of operation days in the system operation cycle. $L_{c u t, i}^{e}, L_{\text {cut }, t}^{h}$ represents the electric load and thermal load of the energy center respectively, $c_{i}^{e}, c_{i}^{h}$ is the energy shortage cost of unit electric load and unit thermal load respectively.

\subsection{Constraints}

On the whole, the constraints of IES control and optimization can be divided into two categories: maximum load constraints and investment cost constraints.

\section{Development trend of IES control}

The current development trend of IES control can be summarized as follows: the control scheme based on CCHP system in structure and the plan scheme with source load uncertainty considered in non-structure.

\subsection{CCHP based control}

In CCHP system, the by-product heat can reach $60-80 \%$ of the total primary energy, while when IES and CCHP are combined, the integration efficiency can reach more than $80 \%$.
In recent years, more and more researches have been done on and CCHP. Literature [8] proposes a hybrid integer programming model which can simulate the IES with CCHP, and integrates the system security constraints into the optimal scheduling model. A model of regional integrated energy system optimization control based on CCHP is proposed in literature [9]. CCHP system is used as interface node to carry out multi-stage multi stage multi plan control and analysis, as well as system location and capacity selection. It can be said that the IES control based on CCHP is the most potential and the most promising direction in the future.

\subsection{Control Considering Uncertainty}

Compared with single energy system, there are many different forms of electrical thermal coupling in IES, and with the increase of distributed power application proportion, the IES has more uncertainty.

In order to solve the uncertainty in IES system and ensure high-quality energy supply, the integrated energy microgrid control with heat and cold is studied in literature [10]. A matrix optimization model is proposed in literature [11] to plan IES. These researches effectively utilize various optimization techniques to solve the uncertainty problems in control.

The analysis shows that the uncertain factors in power system mainly exist in the process of energy supply, transformation and consumption. In this chapter, the uncertain factors of the system are further summarized as load side and energy supply side.

Uncertainty of energy supply side:

The sources of uncertain factors on the energy supply side mainly include three points. First, the random and fluctuating effects of the natural factors such as meteorological conditions on the system output are the first. Secondly, the total amount of surplus energy and the development cost will inevitably change with the development of energy technology. Finally, many external factors, such as market and economic development level, will bring uncertainty to the energy supply side of the system.

In this paper, we develop a mixed integer programming scheme with interval parameter opportunity constraints to solve the problem of high uncertainty and solve the complex problems of control IES under multiple uncertainties. A mixed integer linear programming model with double interval is proposed in literature [12] and applied to the IES control considering reducing greenhouse gas emissions. The paper proposes a comprehensive fuzzy stochastic optimization model developed by combining multi-stage stochastic programming and interval fuzzy analysis technology. The existing methods of randomness and fuzziness analysis are improved, and more uncertain information can be incorporated into its modeling framework.

Load side uncertainty: Most of the existing researches focus on the optimization control of IES energy management / supply side, to some extent, ignore the impact of user demand side on the overall control plan, while there is uncertainty of total cold, hot and 
power demand caused by the different energy demand on the load side. Therefore, the behavior of demand side will significantly affect the control results, and obviously, the uncertainty of demand side is included in the control. The scope of control will become a trend in the future.

The paper introduces an active / passive demand response, which considers the active price response of users to power and passive maintenance of stable system operation. The paper [13] considers the stimulation of demand response, uses the price load model to determine the fuzzy response, and transforms it into the deterministic optimization problem based on the fuzzy programming theory. In literature [14], a user response model considering the uncertainty of user participation is proposed. Network uncertainty represents the relationship between user participation and network incentive.

At present, the research only considers the influence of single side when considering the uncertainty factors, and the research on the dual uncertainty of the supply and use side is relatively few, while the IES control considering the dual uncertainty of source and load side is the key and difficult point of future research.

\section{Conclusion}

This paper first analyzes and summarizes the research on the coordinated control of comprehensive energy system at home and abroad, and obtains the content framework, operation scheduling optimization framework and its internal relations. Secondly, the most widely used objective functions and constraints in the current research are summarized, and a comprehensive energy system control model covering the "network" and "load" storage ring section is obtained. Finally, through the analysis of the related research in recent years, the future general control direction is summarized, including the control scheme based on the cogeneration system and the overall consideration of the source load uncertainty, and makes a reasonable prospect for the future of IES control.

\section{References}

1. Wang J J, Jing Y Y, Zhang C F. Optimization of capacity and operation for CCHP system by genetic algorithm[J]. Applied Energy, 2010, 87(4): 13251335.

2. Carvalho M, Serra L M, Lozano M A. Optimal synthesis of trigeneration systems subject to environmental constraints[J]. Energy, 2011, 36(6): 3779-3790.

3. Zhou Z, Liu P, Li Z, et al. An engineering approach to the optimal design of distributed energy systems in China[J]. Applied Thermal Engineering, 2013, 53(2): 387-396.

4. Rees M, Wu J, Awad B. Steady state flow analysis for integrated urban heat and power distribution networks[C]//2009 44th International Universities Power Engineering Conference (UPEC). IEEE, 2009: 1-5.
5. Wu T, Yang Q, Bao Z, et al. Coordinated energy dispatching in microgrid with wind power generation and plug-in electric vehicles[J]. IEEE Transactions on Smart Grid, 2013, 4(3): 1453-1463.

6. Khodayar M E, Wu L, Shahidehpour M. Hourly coordination of electric vehicle operation and volatile wind power generation in SCUC[J]. IEEE Transactions on Smart Grid, 2012, 3(3): 1271-1279.

7. Basu A K, Bhattacharya A, Chowdhury S, et al. Planned scheduling for economic power sharing in a CHP-based micro-grid[J]. IEEE Transactions on Power systems, 2011, 27(1): 30-38.

8. Geidl M, Andersson G. Optimal power flow of multiple energy carriers[J]. IEEE Transactions on power systems, 2007, 22(1): 145-155.

9. $\mathrm{Li} \mathrm{Z}, \mathrm{Wu} \mathrm{W}$, Shahidehpour M, et al. Combined heat and power dispatch considering pipeline energy storage of district heating network[J]. IEEE Transactions on Sustainable Energy, 2015, 7(1): 1222.

10. Linares P, Romero C. A multiple criteria decision making approach for electricity planning in Spain: economic versus environmental objectives[J]. Journal of the Operational Research Society, 2000, 51(6): 736-743.

11. Antunes C H, Martins A G, Brito I S. A multiple objective mixed integer linear programming model for power generation expansion planning[J]. Energy, 2004, 29(4): 613-627.

12. Chakraborty M, Gupta S. Fuzzy mathematical programming for multi objective linear fractional programming problem[J]. Fuzzy sets and systems, 2002, 125(3): 335-342.

13. Zhu H, Huang $\mathrm{W} \mathrm{W}$, Huang $\mathrm{G} \mathrm{H}$. Planning of regional energy systems: An inexact mixed-integer fractional programming model[J]. Applied Energy, 2014, 113: 500-514.

14. Wu D W, Wang R Z. Combined cooling, heating and power: A review $[\mathrm{J}]$. progress in energy and combustion science, 2006, 32(5-6): 459-495. 